\title{
Context-aware Data Operation Strategies in Edge Systems for High Application Performance
}

\author{
Tanmoy Sen and Haiying Shen \\ Department of Computer Science, University of Virginia, USA \\ \{ts5xm,hs6ms\}@virginia.edu
}

\begin{abstract}
Applications running in edge computing system seamlessly collect data, process data and take actions accordingly. In many cases, the applications need to assist people in real time, and even have lifeor-death consequences such as heart attack detection in healthcare and object detection in driving, which requires low job latency. Moreover, power and bandwidth are constrained resources in edge computing systems. Therefore, a challenge is how to handle data efficiently to reduce job latency, and meanwhile reduce power and bandwidth consumption. Previous works mainly focus on where to store collected source data to reduce the communication latency for source data sharing. Noticing that intermediate and final processing results may be shared by many applications, we propose to store intermediate and final results for sharing to avoid the duplicated computation. We also propose data collection that reduces data collection frequency based on context-related factors to achieve an optimal tradeoff between the overhead and decision making accuracy. We further propose data redundancy elimination to reduce the redundant data transmitted between edge and fog nodes. Our combined data operation strategies show significant improvement over the state-of-the-art methods in terms job latency, power and bandwidth consumption for experiments on both simulated and real edge environment.
\end{abstract}

ACM Reference Format:

Tanmoy Sen and Haiying Shen. 2021. Context-aware Data Operation Strategies in Edge Systems for High Application Performance. In 50th International Conference on Parallel Processing (ICPP '21), August 9-12, 2021, Lemont, IL, USA. ACM, New York, NY, USA, 10 pages. https://doi.org/10.1145/3472456. 3472481

\section{INTRODUCTION}

The rapid increase in the usage of the Internet of Things (IoT) devices and machine learning techniques are leading to a future of using Intelligent Cognitive Assistants (ICA). ICA applications (i.e., jobs) augment human performance and productivity due to their context-aware and adaptive decision-making according to the gathered information from the environment. ICA applications can assist people in working, learning, transportation, healthcare, and other activities.

Permission to make digital or hard copies of all or part of this work for personal or classroom use is granted without fee provided that copies are not made or distributed for profit or commercial advantage and that copies bear this notice and the full citation on the first page. Copyrights for components of this work owned by others than ACM must be honored. Abstracting with credit is permitted. To copy otherwise, or republish, to post on servers or to redistribute to lists, requires prior specific permission and/or a fee. Request permissions from permissions@acm.org.

ICPP '21, August 9-12, 2021, Lemont, IL, USA

(C) 2021 Association for Computing Machinery.

ACM ISBN 978-1-4503-9068-2/21/08 . .\$15.00

https://doi.org/10.1145/3472456.3472481

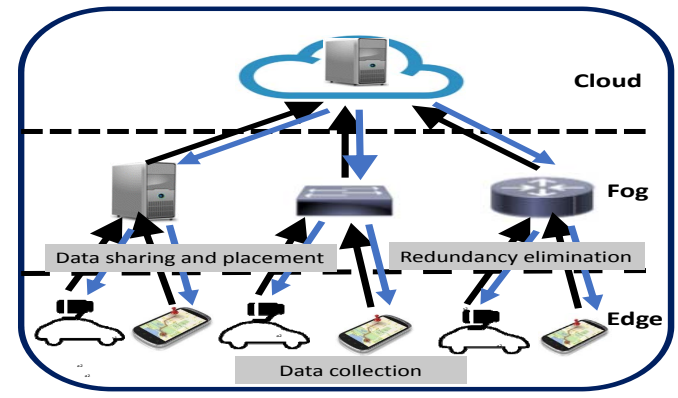

Figure 1: CDOS system overview.

Meanwhile, edge computing systems (consisting of IoT devices, fog and cloud) have emerged as great support for ICA applications. The ICA applications seamlessly collect data, process data and take actions accordingly [7, 31,33]. The application jobs can be scheduled to run in the local edge nodes, fog or cloud to reduce computation and communication overhead [3, 4, 9, 12, 14-16, 24, 26, 29, 30]. Unlike cloud computing that stores and processes data in remote and centralized datacenters, which generates certain delay due to data transmission, edge computing can process data in edge nodes locally. In many cases, ICA needs to assist people in real time, and some even have life-or-death consequences [19]. For example, healthcare ICA in a smart home must be able to predict the heart attack of the resident in time and notify the patient or his/her doctor quickly. An autonomous car must be able to detect a person crossing the street in front of the car in real time. Also, edge nodes such as smartphones and tablets are constrained by the power and the bandwidth due to high network traffic. Therefore, among many challenges in leveraging the edge computing to realize future ICA applications, one challenge is to achieve low job latency with low power and bandwidth consumption.

To handle this challenge, we focus on data operations (including data collection, data placement, and data transmission) in this paper since data communication and data processing contributes significantly to the job latency, power and bandwidth consumption. Compared to task offloading, a little research has been dedicated to studying the data operations in edge computing [25]. In recent years, a few data placement methods $[11,17,18,20,28]$ have been proposed to reduce communication latency for source data sharing since different jobs may share certain source data. Also, there has been a few works $[1,2,32,34]$ that individually focus on selective source data collection for serving the same purpose. Our work considers multiple data operations such as intermediate processing result placement, data collection, and data transmission to reduce not only the communication latency, but also the job latency, power consumption and bandwidth consumption. Specifically, we innovatively leverage the features in the data operations in edge computing for ICA applications to propose a Context-aware Data 
Operation System (CDOS) that incorporates three strategies (shown in Figure 1) accordingly as presented below.

(1) Data sharing and placement.

- Challenge. Many jobs collecting data from the environment and processing collected source data generate high power consumption. Data sharing between edge nodes also consumes high bandwidth It is a challenge to reduce the amount of data to be processed and transferred to reduce the power,bandwidth consumption and job latency.

- Rationale. In addition to the collected source data, intermediate data processing results may be shared by many jobs. For example, in the transportation service, predicted road traffic data is needed by the route recommendation job and optimal velocity calculation job; in the healthcare service, detected breathing rate abnormality is needed for heart attack and asthma attack prediction.

- Strategy. We propose to store intermediate and final computation results needed by many jobs for sharing to avoid duplicate computation and also reduce the amount of transferred data (compared to source data sharing). We formulate a linear programming problem with aim to minimize communication overhead and latency.

(2) Context aware data collection.

- Challenge. The collected data is used for decision making for a job objective (e.g., whether the car will hit a pedestrian). A challenge here is to reduce the amount of data (and hence in data transmission and processing and finally reduce job latency, power consumption and bandwidth consumption) without compromising the decision making accuracy.

- Rationale. Based on the collected data from the environment, each job estimates or predicts (prediction and estimation are interchangeable terms in this paper) a certain event. First, if a collected data item's value (e.g., traffic volume) changes abruptly, it needs to be collected more frequently for closer monitoring and vice versa. Second, if an event is predicted to happen (e.g., traffic congestion), more data for the inputs is needed to have close monitoring for accurate prediction. Third, different input factors have different weights on determining the occurrence of an event, and different events have different importance. For example, the car-pedestrian distance has a higher weight than the traffic volume in determining whether the car will hit the pedestrian, and this event is more important than traffic congestion prediction. Fourth, an event may be more likely to occur in a certain context, e.g., car accident is more likely to occur in heavy traffic in a rainy day, hence needs higher data collection frequency for closer monitoring. Therefore, we can reduce the data collection frequency for the data that is less important in event prediction and monitoring for less important events and vice versa, which can lead to minimization of latency and power consumption. - Strategy. To consider the context in the data collection and reporting, if a situation (e.g., traffic volume) is constant over time, the data collection can be in a lower frequency. If an event (e.g., traffic congestion) is predicted to occur or the context that it is more likely to occur is happening, then the data for the factors affecting the event needs to be collected more frequently for closer monitoring. Further, since different factors have different weights in predicting an event and different events have different weights in the system, the data collection frequency should be determined by the weights. (3) Data redundancy elimination.
- Challenge. With data sharing, nodes (edge, fog and cloud nodes) need to transmit data between each other. These processes generate high bandwidth overhead and long communication delay in network congestion. The challenge here is to reduce the amount of transmitted data without compromising computation accuracy.

- Rationale. In many cases, when an edge node transmits its sensed data (e.g., for environment sensing), there exists much data redundancy in the data stream. The data from many edge nodes may have common data. When a data host transfers data to a data requester, the data stream may contain data in the previously transmitted data, or the data stream itself may contain much redundancy. For example, the temperature keeps almost constant during a certain time period. Geographically close nodes sense similar data from their environment. In this case, traffic redundancy elimination can be used to reduce the amount of data to be transmitted, thus reducing power and bandwidth consumption and job latency (due to shorter data fetching latency).

- Strategy. We apply a redundancy elimination method to avoid transmitting redundant data between edge, fog, and cloud nodes, thus reducing power consumption and latency caused by data transmission.

Our extensive experimental results show that CDOS achieves $55 \%$ improvement on job latency, $46 \%$ on bandwidth utilization and $29 \%$ improvement on energy consumption over the state-of-the-art methods, and also each strategy is effective in reducing job latency, bandwidth, and power consumption. Besides, we also create a small scale real edge test-bed comprising of 5 Raspberry-Pis. On that test-bed, CDOS achieves $26 \%$ improvement on job latency, $29 \%$ improvement on bandwidth utilization and $21 \%$ improvement on power consumption.

The rest of the paper is organized as follows. Section 2 presents the related work. Section 3 and Section 4 presents the detailed design of CDOS and the experimental evaluation of CDOS in comparison with other methods, respectively. Finally, Section 5 concludes the paper with future work.

\section{RELATED WORK}

In the state-of-the-art work for edge computing systems, many approaches [3, 4, 9, 12, 14-16, 26, 29] have been proposed on task scheduling or computation offloading with objectives of reducing various combinations of latency, bandwidth and energy consumption. However, as indicated in [25], little research has been dedicated to studying the data operations and data sharing in edge computing though data operations contribute significantly to job latency, bandwidth, and energy consumption. There are some existing works on data placement $[11,17,18,20,28]$ for edge computing systems. Islam et al. [18] proposed a data placement strategy named iFogStor that is the optimal solution of determining data hosts with the objective of minimizing the upload latency from data generators to the data hosts and from the data hosts to data consumers (the devices that require the data). Based on this work, the authors further proposed iFogStorG [17], which finds the optimal solution on the partitioned graph rather than the whole set of devices as in iFogStor. The authors defined vertex weight of a node as the number of dataitems in that particular node plus one, and defined edge weight as the number of data flows passing through the related physical link, and used graph partitioning that considers vertex weights to 
balance data items and data hosts between partitions, and considers edge weights to minimize inter-partition data flows. The partitions are disjoint, and data is placed in a node that has lower overall latency (upload and download time) within each partition. Rashid et al. [20] proposed Edgestore, which is an edge-based distributed storage system for emergency response. Zeng et al. [28] considered a fog computing supported software-defined system, where job images (i.e., saved states of running jobs) lay in the storage servers to avoid re-computation upon failures. For data sharing in edge computing systems, quite a few approaches $[8,21,23]$ have been proposed for caching shared data in the edge nodes.

Several methods $[1,2,32,34]$ have been proposed to determine how to decrease the transmitted data samples without increasing the estimation error in the IoT or cyber-physical systems. Our work is different in that it focuses on considering multiple contextrelated factors in reducing data sampling frequency. He et al. [6] proposed traffic redundancy elimination (TRE) technique named TailoredRE that leverages the cloud resource to create clones to offload the overhead of elimination from smartphones. Yu et al. [27] proposed a Cooperative end-to-end TRE solution (CoRE) that can detect and remove both short-term redundancy (repetition in minutes) and long-term redundancy (repetition in hours or days) with cooperative operations between the data sender and receiver in the cloud environment to reduce its bandwidth cost. Unlike these works, our work studies the effectiveness of applying the TRE techniques in the edge computing systems to reduce the bandwidth and energy overhead on edge nodes.

\section{SYSTEM DESIGN OF CDOS}

\subsection{Assumptions}

We present the assumptions of this work below.

- Each job instance is deployed to a specific edge node by a job scheduling algorithm $[4,9,12,15,24,29]$.

- The edge computing system runs pre-defined jobs and the jobs will not change during a certain time period (e.g., 1 hour). Therefore, the system has information regarding the source data needed by each job, dependency between different jobs. This dependency means that a job needs the data collected or generated by another job.

- The system has a server (called data placement scheduler) in the cloud or the fog to conduct the data placement scheduling based on the dependency of the jobs.

- The system has predefined priorities for the jobs (or predicted events) based on their job objectives. For example, predicting the occurrence of a car accident has a higher priority than predicting road congestion.

- We cluster geographically close edge nodes in an area together (called geographical cluster) for data sharing, and the nodes in a geographical cluster remain same in a certain time period and can communicate with each other.

In the following, we present the three strategies of CDOS as shown in Figure 1.

\subsection{Data Sharing and Placement}

As explained previously, edge nodes need to collect the same data and may also need to calculate intermediate results. Some edge nodes' intermediate results may be the intermediate results or final results of other edge nodes. For example, as shown in Figure 2, the

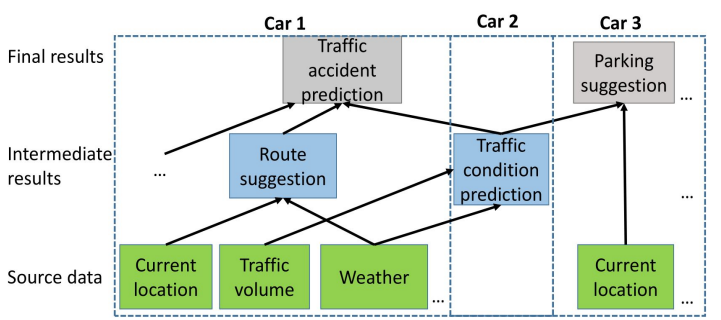

Figure 2: Sharing source, intermediate and final data among jobs.

weather source data is collected by many edge nodes for their different jobs such as parking suggestion, traffic condition prediction, and traffic accident prediction. The final results of traffic condition prediction from car2 can be used as the intermediate results in car1 (for traffic accident prediction) and the intermediate results for car3 for parking suggestion. Therefore, edge nodes in a geographical cluster can share source data and computation results to avoid duplicated data collection and computation, thus reducing both power consumption and computation latency.

Data sharing brings about a problem on where the shared data should be stored so that each edge node can fetch its needed data efficiently with low latency and low bandwidth consumption. In our solution to this problem, the data placement scheduler determines the data to be shared based on the job dependency for the next time period, and then solves a linear programming problem to determine the nodes to place the data items and notifies other nodes. The data generators will then store their data to the scheduled data hosts for sharing, and the data requesters will fetch their needed data from the data hosts for their job computation.

As the data placement schedule is calculated proactively before the shared data is generated, the latency for solving the linear programming problem will not affect the job latency. Also, the data placement schedule does not have to be calculated periodically. Instead, as long as the jobs and edge nodes in a geographical cluster do not change, the previously calculated data placement schedule still is valid specifically for Machine Learning models with static execution flow. However, when an edge node has a new job, removes an existing job, moves out of the geographical cluster, or joins in a geographical cluster, it needs to notify the scheduler. Then, only when the number of changed jobs and/or changed nodes reach a certain level that will change the schedule greatly, the scheduler conducts the data placement scheduling again.

3.2.1 Determine Shared Source and Result Data. Each job consists of a few tasks that generate intermediate results. To find the dependency among the data-items and tasks, the scheduler generates a dependency graph as shown in Figure 3. From the dependency graph, the scheduler derives which jobs share which source data, intermediate data and final results, and finally determines the shared data to be stored. The dependency graph may change dynamically depending on the applications. In our case, we assume it keeps constant for a certain period.

3.2.2 Linear Program to Determine Data Hosts. After the data-items to be shared are determined, the scheduler then needs to determine the fog or edge nodes in each geographical cluster to place the 


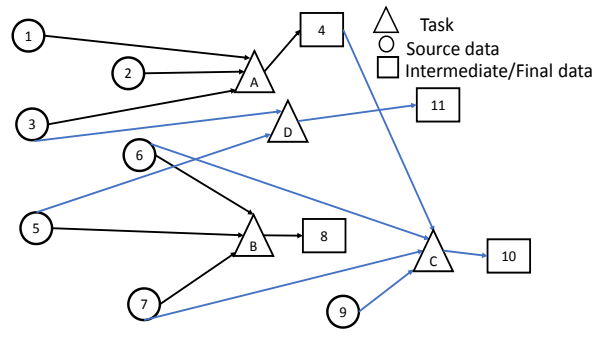

Figure 3: Dependency graph for shared data.

data-items. The nodes within a geographical location may be heterogeneous in terms of storage capacities and network bandwidth between them. If a job needs a data-item, we call it this data-item's dependent job. We aim to place each shared data-item to a node that i) is close to the nodes running its dependent jobs as much as possible, ii) has high network bandwidth to the nodes of its dependent jobs, and iii) has enough storage capacity to store the data-item.

We formulate a linear programming problem for deciding the placement of shared data-items. We use $d_{j}$ to denote data-item $j$, use $s\left(d_{j}\right)$ to denote its size, and use $n$ to denote a node. $c\left(n_{p}, n_{d}, d_{j}\right)$ denotes the network bandwidth cost incurred by moving data-item $d_{j}$ from the node $n_{p}$ to node $n_{d}$. It is calculated by the following equation [13]:

$$
c\left(n_{p}, n_{d}, d_{j}\right)=h\left(n_{p}, n_{d}\right) \cdot s\left(d_{j}\right)
$$

where $h\left(n_{p}, n_{d}\right)$ is the number of hops between $n_{p}$ and $n_{d}$.

We use $l\left(n_{p}, n_{d}, d_{j}\right)$ to denote the time required to transmit dataitem $d_{j}$ from node $n_{p}$ to node $n_{d}$. It is dependent on the bandwidth of the link between the node $n_{p}$ and node $n_{d}$, denoted by $b\left(n_{p}, n_{d}\right)$ and is calculated by:

$$
l\left(n_{p}, n_{d}, d_{j}\right)=s\left(d_{j}\right) / b\left(n_{p}, n_{d}\right)
$$

If data-item $d_{j}$ is sensed or generated by node $n_{g}$ and is scheduled to be placed in node $n_{s}$, and the set of nodes running $d_{j}$ 's dependent jobs is denoted by $N_{d}^{d_{j}}$. Then, the total bandwidth cost for storing data and fetching data is calculated by:

$$
C\left(n_{g}, n_{s}, d_{j}, N_{d}^{d_{j}}\right)=c\left(n_{g}, n_{s}, d_{j}\right)+\sum_{n_{d} \in N_{d}^{d_{j}}} c\left(n_{s}, n_{d}, d_{j}\right)
$$

Similarly, the total latency for storing and fetching data item $d_{j}$ is calculated by:

$$
L\left(n_{g}, n_{s}, d_{j}, N_{d}^{d_{j}}\right)=l\left(n_{g}, n_{s}, d_{j}\right)+\sum_{n_{d} \in N_{d}^{d_{j}}} l\left(n_{s}, n_{d}, d_{j}\right)
$$

We use $N$ to denote the set of all edge and fog nodes that can store data, use $N_{g}$ to denote the set of data generators, $D_{g}$ to denote the generated data-items to be stored, and use $S_{n_{s}}$ to denote the available storage capacity of node $n_{s}$. The scheduler decides the nodes to store the shared data-items by formulating and solving the following linear programming problem:

$$
\begin{aligned}
& \text { Min: } \forall n_{s} \in N^{\forall} d_{j} \in D_{g}, n_{g} \in N_{g} C\left(n_{g}, n_{s}, d_{j}, N_{d}^{d_{j}}\right) . \\
& L\left(n_{g}, n_{s}, d_{j}, N_{d}^{d_{j}}\right) \cdot x\left(d_{j}, n_{s}\right)
\end{aligned}
$$

subject to:

$$
\begin{gathered}
\sum_{d_{j} \in D_{g}} s\left(d_{j}\right) \cdot x\left(d_{j}, n_{s}\right) \leq S_{n_{s}}, \forall n_{s} \in N \\
x\left(d_{j}, n_{s}\right) \in\{0,1\} \\
\sum_{n_{s} \in N} x\left(d_{j}, n_{s}\right)=1, \forall d_{j} \in D_{g}
\end{gathered}
$$

Here, $x\left(d_{j}, n_{s}\right)$ refers whether data-item $d_{j}$ is placed on a node $n_{s}$ or not. The objective of the linear programming problem in Equation (5) is to minimize the incurred bandwidth cost and transmission latency of the generated data-items at a certain time period. Equation (6) ensures that the sum of the sizes of the data-items allocated to a node does not exceed its available storage capacity. Equations (7) and (8) cumulatively ensure that one data-item is placed in only one node.

\subsection{Context aware Data Collection}

If an edge node collects data from the environment at a certain frequency, on the one hand, the collected data may not be enough to achieve high event prediction accuracy, and on the other hand, it may waste power by collecting redundant data such as constant temperature for a long time period. Previous approaches [1, 34] focus on how to decrease the transmitted data samples to avoid network congestion while limiting the prediction or estimation error. Our method in this paper tackles this problem from a different angle; it identifies different context-related factors in determining the data collection (or sampling) frequency. We assume that each job (e.g., event prediction) has a priority and a tolerable prediction error correspondingly. For example, prediction vehicle collision has a higher priority and a lower tolerable prediction error, while traffic condition prediction has a lower priority and a higher tolerable prediction error. Therefore, our goal is to minimize the amount of collected data while guaranteeing that the job computation error is no larger than the tolerable error. In this paper, the following lists our identified factors that can be considered in adjusting the data collection frequency, and other factors can be easily included in our strategy in the same manner.

3.3.1 Abnormality of Data. The sensed data of a data type by an edge can be considered as a time-series. When the sensed data (e.g., temperature, objects in front of a car) is constant and normal over time, the data collection can be in a lower frequency and whenever it shows an abnormality (e.g., sudden change in temperature, sudden appearance of a passenger), it should be collected more frequently to more accurately catch the abnormality for accurate prediction and timely reaction.

For observing data abnormality, each edge node maintains an event-wise statistics consisting of mean (denoted by $\mu$ ) and standard deviation (denoted by $\delta$ ) of the data-items from the historical data. A data value is declared abnormal when it is not within the range of $|\mu \pm \rho \cdot \delta|$. Each edge node processes the time-series data as a sequence of sliding windows. Each sliding window consists of $M$ data-items. Within a sliding window, if an edge node observes such data abnormality for $m(0<m \leq M)$ consecutive times, the edge node declares it as an abnormal situation. We define abnormality parameter $w_{d_{j}}^{1}$ to reflect the abnormality of the type of data-item $d_{j}$ :

$$
w_{d_{j}}^{1}=\left|\left(\sum_{i=1}^{m} v_{i}\left(d_{j}\right) / m-\mu\right)\right| /\left(\rho_{\max } \cdot \delta\right)+\epsilon,
$$

where $v_{i}\left(d_{j}\right)$ is the $i^{t h}$ value of data $d_{j}, \rho_{\max } \cdot \delta$ denotes the maximum standard deviation and $\epsilon(0<\epsilon<1)$ is a very small fraction. $w_{d_{j}}^{1}$ represents the distance of the mean of the abnormal values from the mean of the original distribution. When there is an abnormality detection of $d_{j}$, an edge node updates $w_{d_{j}}^{1}$ using Equation (9). The 
range of $w_{d_{j}}^{1}$ is always $0<w_{d_{j}}^{1} \leq 1$; a higher abnormality leads to a larger $w_{d_{j}}^{1}$ and vice versa. The parameters $\rho$ and $\rho_{\max }\left(\rho<\rho_{\max }\right)$ are set by the system or the edge node. For example, in Gaussian distribution, all values are within $3 \delta$, so we can set $\rho_{\max }=3$ and $\rho=2$.

3.3.2 Priority of Events. In an edge computing system, there are many types of jobs with different priorities, i.e., the requirements on computation/prediction accuracy. For example, car collision prediction has a higher priority than traffic condition prediction. As we assumed, the system assigns different jobs with different priorities. The priority of an event/job $\left(e_{i}\right)$ is denoted by parameter $w_{e_{i}}^{2}$ $\left(0<w_{e_{i}}^{2} \leq 1\right)$. Such a priority list is copied and stored in the edge nodes. Also, when an event is predicted to occur with a certain probability (denoted by $p_{e_{i}}$ ), data related to the event should be collected more frequently to confirm whether the event will occur or not with higher accuracy. Combining these two considerations, each edge node periodically updates the priority parameter of event $e_{i}$ by $w_{e_{i}}^{2}=w_{e_{i}}^{2} \cdot\left(p_{e_{i}}+\epsilon\right)$.

3.3.3 Data Weight on Computation Result. All the data-items do not equally contribute to the occurrence of an event. Data-items that have more contribution on the occurrence of an event should be collected more frequently and vice versa. For example, the time of a day has a higher weight than the temperature in the traffic condition prediction. Machine learning is a widely used method for event prediction, and the machine learning model (such as Bayesian network) determines the weights of inputs on the predicted event, denoted by $p_{d_{j}, e_{i}}$. We define the data weight parameter of data-item $d_{j}$ on event $e_{i}$ by $w_{d_{j}, e_{i}}^{3}=p_{d_{j}, e_{i}}+\epsilon\left(0<w_{d, e_{i}}^{3} \leq 1\right)$. As shown in Figure 2, a job may consist a sequence of tasks in the hierarchical structure with intermediate results $\left(e_{i}\right)$ for computing the final results $\left(e_{k}\right)$. In this case, $w_{d_{j}, e_{k}}^{3}=w_{d_{j}, e_{i}}^{3} \cdot w_{e_{i}, e_{i+1}}^{3}, \ldots ., \cdot w_{e_{k-1}, e_{k}}^{3}$, where $e_{i}, e_{i+1}, \ldots, e_{k-1}$ denote the intermediate results of different layers in hierarchical structure.

3.3.4 Context of an Event. The context of an event may affect the probability of the event occurrence. For example, car accidents are more likely to occur in heavy traffic in rainy weather than in light traffic in sunny weather. Therefore, the input data for car accident prediction needs to be collected more frequently in heavy traffic in rainy weather than in light traffic in sunny weather. Taking this into account, we adjust the data collection frequency of data-items according to the context of the event. Based on the practical knowledge, the system specifies the contexts for each event/job in which the input data-items of the event need to be more frequently collected. The context is expressed by the value ranges of the source data-items, the intermediate results, and the final results. We use $w_{e_{i}}^{4}=\sum_{k} w_{c_{i, k}}^{4}+\epsilon\left(0<w_{e_{i}}^{4} \leq 1\right)$ to denote the probability that the specified contexts of event $e_{i}$ is true, where $w_{c_{i, k}}^{4}$ denotes the probability of specified context $k$ of event $e_{i}$ is true.

3.3.5 Adaptive Data Collection Frequency Adjustment. Finally, we determine the final weight for a data-item. If data-item $d_{j}$ is the input of a set of multiple events/jobs $\left(E_{j}\right)$, its final weight equals:

$$
W_{d_{j}}=\sum_{e_{i} \in E_{j}} w_{d_{j}}^{1} \cdot w_{e_{i}}^{2} \cdot w_{d_{j}, e_{i}}^{3} \cdot w_{e_{i}}^{4},\left(0<W_{d_{j}} \leq 1\right)
$$

The final weight of each data-item, $d_{j}$, in the computation is used to tune the data collection frequency of $d_{j}$. Collecting data at a lower frequency causes the edge node to have high computation error or miss certain events and vice versa. We aim to set the collection frequency of a data-item so that the prediction errors of its dependent jobs are limited to their tolerable prediction errors. The prediction error is measured by the percentage of the incorrect predictions among all predictions.

The additive linear increase multiplicative decrease (AIMD) algorithm $[10,22]$ is a feedback control algorithm best known for its use in TCP congestion control. AIMD combines linear growth of the congestion window with an exponential reduction when congestion is detected. Similarly, we use AIMD to adjust the data collection time interval (i.e., the reciprocal of data collection frequency) based on the prediction errors of data $d_{j}$ 's dependent jobs as below:

$$
T_{t+1}= \begin{cases}T_{t}+\alpha / \eta W_{d_{j}}(\alpha \geq 1), & \text { all errors are within their limits } \\ \frac{T_{t}}{\beta+\eta W_{d_{j}}}(\beta \geq 1), & \text { otherwise. }\end{cases}
$$

where $\eta$ is used to adjust the weight of data $d_{j}$ to achieve the best performance. When the errors of the data-item's dependent jobs are within their limits, data collection frequency can be reduced, so the time interval increases additively by $\alpha / \eta W_{d_{j}}$. The higher the weight, the lower the increase of updated time interval, which reciprocally limits the decrease of collection frequency and vice versa. When the error is beyond the limit, data collection frequency should be increased, and the time interval decreases multiplicatively by $\beta+\eta W_{d_{j}}$. The higher the weight, the higher the decrease of updated time interval. Therefore, the data for higher priority events have lower decrease in data collection time interval.

\subsection{Data Redundancy Elimination}

In the edge computing system, the jobs may be allocated to edge, fog, and cloud nodes. Before an edge node, fog, or cloud executes a job, it needs to fetch the data-items needed for the computation, and then transmits results back to the nodes that need the computation results. In many cases, the sensed datasets (e.g., for environment sensing) at different times by the edge nodes in the same geographical location have significant duplication. For instance, many autonomous cars on the same road collect the same data and transmit the data to the fog or cloud. Also, the environmental data in different time slots in a long time period may not change greatly. In such scenarios, sending such redundant data to the fog or cloud induce unnecessary bandwidth consumption of the network as well as power consumption of the edge nodes. Using our data sharing and placement strategy, there will be more data transfer operations and hence more redundant data in transmission. Thus, elimination of redundant data in data transmission between edge nodes, fog and cloud help to reduce the amount of data to be transmitted, which results in the reduction of power, bandwidth and data transmission latency. Accordingly, we use a data redundancy elimination strategy based on prior proposed CoRE [27] that is for the cloud environment. Rather than focusing on the data transfer from the cloud to a client, in our edge computing system, the redundancy elimination strategy is used by a pair of data sender and data receiver that always transfer data between themselves, and the data transfer directions can be in both directions between two edge nodes, two edge-fog nodes, and two edge-cloud nodes. 


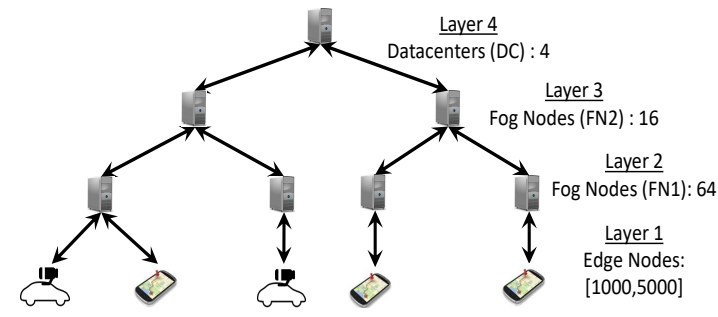

Figure 4: A high-level edge computing architecture. 4 EXPERIMENTAL EVALUATION

\subsection{Experimental Setup}

To evaluate the performance of CDOS, we used iFogSim [5] to simulate our customized edge/fog computing environment. We updated iFogSim to support our data placement, data collection, and redundancy elimination strategies.

Edge computing architecture related settings. Our experiment was conducted on a four-layer edge-fog-cloud architecture involving edge nodes, fog devices, and cloud data centers [18] as shown in Figure 4. We performed the simulation using 4 data centers (DC), 16 fog nodes in layer 1 (FN1), and 64 fog nodes in layer 2 (FN2). We varied the number of edge nodes from 1000 to 5000 with 1000 increase in each step. We grouped all of these nodes into four geographical clusters, where each cluster contains an equal number of nodes from every layer. All other important simulation parameters are indicated in Table 1 . We randomly chose a value from the specified range for the setting.

Data and job related settings. In the system, there were 10 types of source data to be sensed and 10 types of jobs. As in [17], to generate the values of each type of source data, we used one Gaussian distribution of mean randomly selected from $[5,25]$ and standard deviation randomly selected from $[2.5,10]$. The edge nodes collected the source data with a default collection frequency of 1 data-item per $0.1 \mathrm{~s}$. This collection frequency was later tuned based on our context aware data collection strategy in each time window of $3 \mathrm{~s}$. To test the redundancy elimination performance even when data chunks are not completely the same, for each data-item stream generated by a Gaussian distribution, we randomly chose 5 data-items from each window of 30 data-items, and then changed one random byte at a random position as in [27] to reflect the subtle change in the environment. We set the size of the chunk-cache to $1 \mathrm{MB}$.

Each type of job needs $x$ number types of data-items and $x$ is randomly chosen from $[2,6]$. Each job generates two intermediate results and one final result data-item, which can be shared among nodes. For each type of jobs, we build a hierarchical structure (see Figure 2) to generate the dependency among its sensed source dataitems, intermediate and final data-items. The same input data-items generate the same output intermediate and final data-item. Each node is randomly assigned with a job. Each node runs its job every 3s. Therefore, in a geographical cluster, based on such hierarchical structures, we can find nodes that share source, intermediate and final data-items. Among the nodes that share the same data, we randomly chose one node to sense or calculate the intermediate or final data-items to share. Here, the size of one type of input source data (without collection frequency adjustment), intermediate and final data-item was set to $64 \mathrm{~KB}$.
We assigned the 10 types of jobs with priority scores from [0.1, $0.2, \ldots, 1]$ in sequence. We set the tolerable prediction errors for the different events/jobs with a value from range [1\%, 5\%]; jobs with priority $0.1-0.2$ have tolerable error of $5 \%$, jobs with priority 0.3-0.4 have tolerable error of $4 \%$ and so on. We considered each type of jobs as an event prediction and built a Bayesian network for computing an intermediate result or a final result. We assumed that the latency for each task computation increases proportionally to the amount of input data; $64 \mathrm{~KB}$ data taking $0.1 \mathrm{~s}$ to be processed.

For training a Bayesian network for event prediction, we generated synthetic data. Specifically, we divided the distribution of each input data-item into random non-overlapping ranges. We used each combination of the ranges of all input data-items to represent a context and randomly selected two contexts as the specified contexts that the event was occurring. Also, when one source data is in abnormal ranges, we always set the output as 1 . We associated other contexts to the output 1 (event is occurring) or 0 (event is not occurring) randomly. We consider this generated training data as the ground truth. We collected the experimental results after running 16 hours. We ran each experiment for 10 times. We set $\rho_{\max }=3, \rho=2$ due to the reason explained before. We also set $\alpha=5, \beta=9$, and $\eta=1$ as these values achieve the best performance in our experiments.

\subsection{Compared Methods}

- iFogStor [18]: It finds data hosts (among edge and fog nodes) using linear programming which minimizes overall data transmission latency (i.e., latency to transfer data from source to host and host to consumers) while satisfying the storage capacity constraints of the nodes.

- iFogStorG [17]: iFogStorG partitions the fog infrastructure in several sub-graphs and finds the optimal data placement solution on the partitioned graph, as explained in Section 2. Using graph modeling and partitioning approach, the authors first created several sub-problems, and then used a divide and conquered heuristic to find host nodes from those sub-problems, which requires the least communication latency to store and retrieve data.

- LocalSense: In this approach, each edge node senses all of its needed source data-items for its computation jobs. We did not set storage capacity limit in this method to measure the job latency performance of CDOS (with data fetching latency) in comparison with the method with the shortest job latency without using our strategies.

We compare these methods with our individual data operation strategies as well as the combined CDOS using the mentioned experiment settings for simulated and real environment.

\subsection{Performance Metrics}

- Job latency: It is the time period from the time to fetch data for computation to the time when the job completes.

- Bandwidth utilization: We traced the overall bandwidth required to perform data collection, data placement, and data retrieval of edge nodes from the simulator iFogSim [5].

- Consumed energy: This is the total energy consumed by the edge nodes for data collection, computation and retrieval.

- Frequency ratio: It is the ratio between the current collection frequency and the default original collection frequency for the data-items. 
Table 1: Simulation parameters.

\begin{tabular}{ccccc}
\hline \multicolumn{2}{c}{ Edge node (EN) } & \multicolumn{2}{c}{ Fog node (FN1 \& FN2) } \\
\cline { 1 - 2 } \cline { 4 - 5 } Storage capacity & $10 \mathrm{MB}-200 \mathrm{MB}$ & Storage capacity & 150MB- 1GB \\
\hline Edge-FN1 network bandwidth & $1 \mathrm{Mbps}-2 \mathrm{Mbps}$ & & FN1-FN2 network bandwidth & $3 \mathrm{Mbps}-10 \mathrm{Mbps}$ \\
\hline Idle/Busy power & $1 / 10 \mathrm{MW}$ & & Idle/Busy power & $80 / 120 \mathrm{MW}$ \\
\hline
\end{tabular}

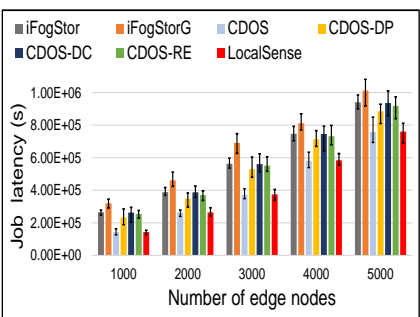

(a) Job latency

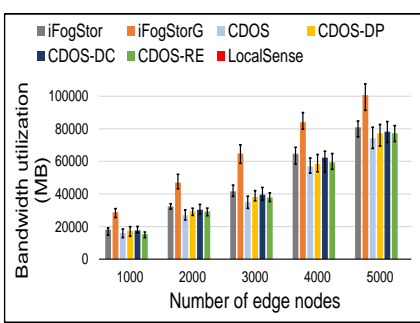

(b) Bandwidth utilization

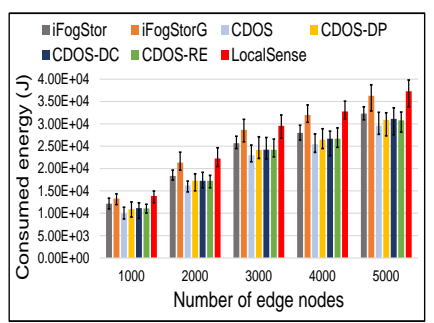

(c) Consumed energy

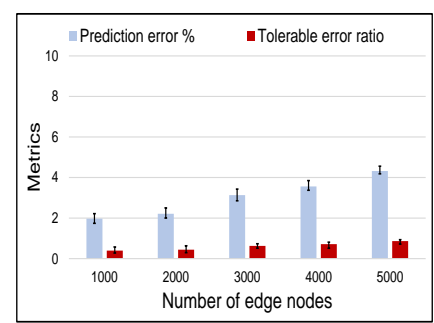

(d) Prediction error

Figure 5: Overall performance comparison and effectiveness of each strategy in CDOS.

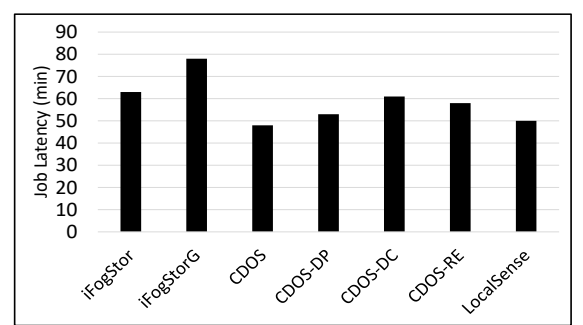

(a) Job latency

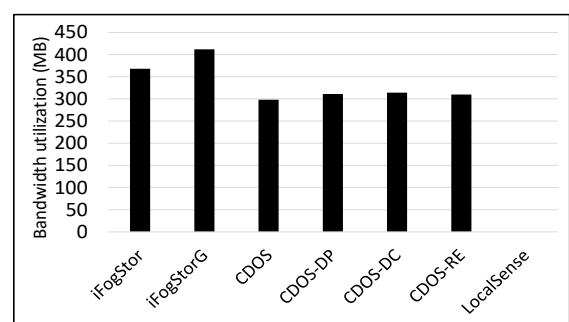

(b) Bandwidth utilization

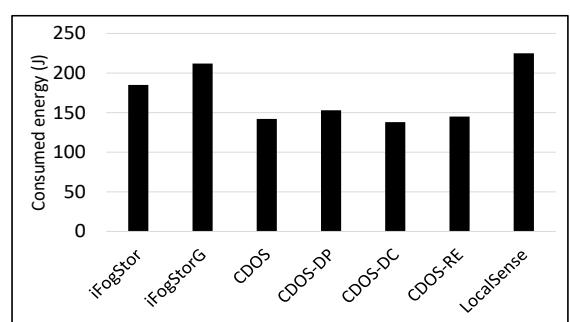

(c) Consumed energy

Figure 6: Overall performance comparison and effectiveness of each strategy in CDOS for real devices.

- Prediction error: It is calculated as the percentage of times that fail to detect an event accurately.

- Tolerable error ratio: It is the ratio of prediction error to the tolerable prediction error and it measures whether the prediction error is within the tolerable error.

\subsection{Experimental Results}

4.4.1 Overall Performance. Figure 5 illustrates the overall performance of CDOS, iFogStor, IFogStorG and LocalSense in terms of total job latency, bandwidth utilization, consumed energy in Joules (J) and prediction accuracy versus the number of edge nodes. The results illustrate the mean, the $5 \%$ and $95 \%$ percentiles of the ten experiment runs. We also include the performance of each strategy of CDOS, including data sharing and placement (denoted by CDOS-DP), data collection (denoted by CDOS-DC) and redundancy elimination (denoted by CDOS-RE). The data placement in CDOSDC and CDOS-RE was built upon iFogStor.

From the figure, we see that iFogStorG always performs worse compared to iFogStor. This is because to determine the data placement schedule, iFogStorG uses a heuristic-based approach while iFogStor solves the optimization problem. We calculate the improvement by $|x-\hat{x}| / x$, where $x$ and $\hat{x}$ are the performance measures of the compared method and our method respectively. Compared to iFogStor, CDOS achieved 23\%-55\% improvement on job latency, 21\%$46 \%$ improvement on bandwidth utilization and $18 \%-29 \%$ improvement on energy consumption. This improvement is the outcome of the combination of systematically addressing the data collection, data placement and redundancy elimination issues.

For job latency, as CDOS enables sharing the intermediate and final result data, nodes can avoid repetitive calculation and directly use the intermediate and final computation results from other nodes. The data can be fetched quickly since one goal of the solution of the optimization problem for data placement is to minimize the data fetch latency. Also, the amount of shared source data is reduced due to the data collection strategy. The redundancy elimination strategy further reduces the amount of data to be fetched and hence reduces the data fetching latency. In addition, the solution of the data placement is derived in advance before the data is sensed or calculated, so a node stores its sensed data or computation result data to their assigned nodes once the data is available without delay. As a result, CDOS reduces the job latency of iFogStor. The performance of CDOS in terms of job latency is similar to LocalSense, which does not have data fetching delay.

For the bandwidth consumption, CDOS's data collection strategy reduces data sensing frequency accordingly and hence reduces the amount of collected data, its data sharing and placement strategy enables sharing intermediate and final results and hence reduces the amount of fetched source data, and it tries to reduce bandwidth consumption, and its redundancy elimination strategy reduces the amount of data to be transmitted. Consequently, CDOS reduces bandwidth consumption on edge nodes. LocalSense does not have any bandwidth consumption since each edge node does not transmit any data-items and executes all jobs by itself. 
For the energy consumption, in CDOS, an edge node does not have to sense data at the full frequency, it sometimes can get the intermediate or final computation results from other nodes without the need for the computation itself, and also it can reduce the amount of data it receives or sends due to the intermediate and final result sharing as well as the redundancy elimination strategy. Finally, each node's consumed energy can be reduced. LocalSense consumes much higher energy than other methods because it does not have source data sharing and each node must collect all data needed by its computation.

In CDOS, for each edge node, we calculated its average prediction error and tolerable error ratio over the simulation time. Then, we measured the mean, the $5 \%$ percentile, and the $95 \%$ percentile of predic-

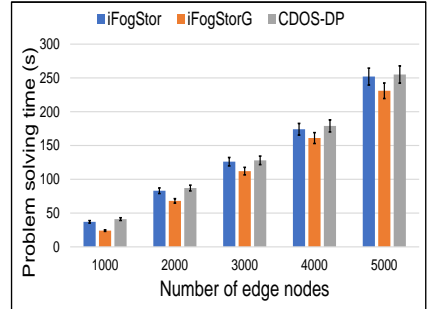

Figure 7: Computation time. tion error and tolerable error ratio and showed the results in Figure $5 \mathrm{~d}$. We do not include other methods here as they do not focus on computation accuracy. We can observe that for different number of edge nodes, the average prediction error is always within the limit of 5\%. We also see that the tolerable error ratio is always within 1 . The results mean that CDOS can always generate low computation errors and satisfy tolerable error requirements of jobs.

Finally, we measured the computation latency for data placement methods in iFogstor, iFogstorG and CDOS-DP in Figure 7. iFogStorG needs $12 \%$ less time than iFogStor and CDOS, which produce similar computation latency. This is because iFogStorG is a heuristic method, while iFogStor and CDOS solve an optimization problem. However, CDOS has a new strategy that only when dataitem change or node change reach certain levels, it reschedules the data placement by solving the optimization problem. Therefore, its number of times to solve the optimization problem is much less than those of iFogstor and iFogstorG. Also, as CDOS conducts the data placement proactively before the data is sensed or generated, the problem solving delay will not bring about a delay for data placement or data fetching for computation.

Moreover, for all the measurements, we observe that the metrics in the y-axis increase as the number of edge nodes increase in the $\mathrm{x}$-axis. This phenomenon occurs because with the increase of the number of edge nodes, the number of data-items and jobs also increase. This increase contributes to additional time, bandwidth and energy for fetching data and completing all jobs, and additional time for solving the data placement optimization problem for a larger-scale system. In addition, the prediction error and tolerable error ratio increase as the number of edge nodes increase because when there are more edge nodes, the probability that an edge node generates a higher prediction error is increased.

4.4.2 Performance on Real Devices. Figure 6 illustrates the overall performance of CDOS, iFogStor, IFogStorG and LocalSense in terms of total job latency, bandwidth utilization, consumed energy on real test-bed. The test-bed consists of 5 Raspberry-Pi-4s, among them, two Pis have 1 GB memory, two other Pis have 2 GB memory and another Pi has 4 GB memory. We also use 2 laptops as fog nodes and 1 remote cloud datacenter. All nodes are connected via $2.4 \mathrm{GHz}$ band wireless connection. Compared to iFogStor, CDOS achieved $26 \%$ improvement on job latency, $29 \%$ improvement on bandwidth utilization and $21 \%$ improvement on energy consumption. The reasons for such improvement is the result of reasons as mentioned above.

4.4.3 Effectiveness of Each Strategy. Figure 5a, Figure 5b and Figure $5 \mathrm{c}$ also show the performance of each individual strategies in CDOS regarding job latency, bandwidth utilization and consumed energy. For job latency, CDOS-DP performs 15-27\% better than iFogStor and iFogStorG but performs slightly worse than LocalSense (1-6\%) due to its additional data fetching time. For bandwidth utilization, CDOS-DP shows $8-27 \%$ improvement over iFogStor and iFogStorG.

For consumed energy, CDOS-DP performs 5-17\% better than all three compared methods. Our data placement strategy smartly enables to share intermediate and final results. It helps reduce job latency in computation and reduce bandwidth utilization and power consumption caused by computation. Also, it helps reduces the amount of fetched source data for computation, thus reducing bandwidth utilization and consumed energy. CDOS-DC improves the job latency, bandwidth utilization, and consumed energy of iFogStor and iFogStorG by a range of $1-23 \%, 3-22 \%$, and $5-29 \%$, respectively. Compared with LocalSense, CDOS-DC performs $16-25 \%$ better in terms of consumed energy and $4-10 \%$ worse in terms of job latency. Our data collection strategy reduces the amount of collected data, so it reduces the consumed energy for data collection. Also, it reduces the amount of data to be fetched and processed, and then reduces job latency, bandwidth utilization, and consumed energy. Its higher job latency compared to LocalSense is caused by data fetching. Compared to iFogStor and iFogStorG, CDOS-RE improves their job latency by $5-13 \%$, and improves the consumed energy of them and LocalSense by $4-16 \%$. Our redundancy elimination strategy reduces the amount of transferred data, and thus reducing job latency, bandwidth utilization, and the consumed energy. While for job latency, LocalSense performs better than CDOS-RE by $2-6 \%$ due to CDOS-RE's additional data fetching time. In conclusion, each strategy in CDOS is effective in reducing job latency, bandwidth utilization, and energy consumption. It only generates a slightly higher job latency due to additional data fetching time. However, without data sharing, LocalSense consumes high energy.

4.4.4 Effect of Context-related Factors in Data Collection in CDOS. Figure 8 plots frequency ratio, prediction error and tolerable error ratio for each context-related factor. We calculated the frequency ratio of an event/job as the average frequency of all of its input data-items in the event prediction. We group the final results with the same factor value in the $\mathrm{x}$-axis and calculated the average value in each group as the y-axis value as the final results. From Figure 8, we observe that as the values of the individual parameters increase in the $\mathrm{x}$-axis, the frequency ratio increases, and the prediction error decreases because of the adaptive change of collection frequency according to the overall weight. In detail, when the factor is more important (i.e., more abnormal datapoints of an event in Figure 8a, higher priority of an event in Figure 8b, higher weights of input dataitems of an event in Figure 8c, or more specified context occurrences in Figure 8d), the data collection frequency are increased for closer monitoring and hence the prediction error decreases, and vice versa. 


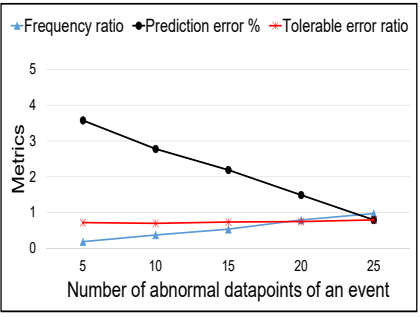

(a) Abnormal datapoints

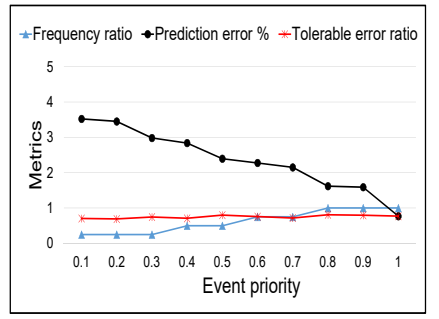

(b) Event priority

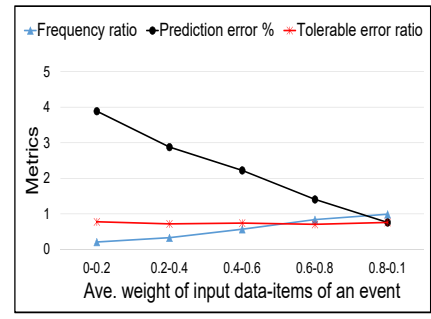

(c) Ave. weight of input data-items

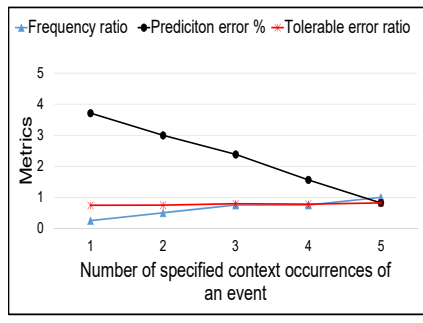

(d) Specified context occurrences

Figure 8: Effect of different parameters on data collection frequency and computation error.

Moreover, for all factors, the tolerable error does not change and is always $<1$.

Figure 9 shows impact of data frequency ratio on the job latency, bandwidth utilization, consumed energy, prediction error and tolerable error ratio in CDOS. We classified the frequency ratio range to $[0,0.2],[0.2,0.4] \ldots[0.8,1]$. We grouped the results of job latency, node bandwidth utilization, node

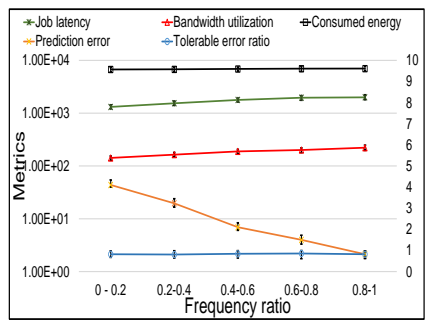

Figure 9: Metrics for frequency ratio. consumed energy, job prediction error and tolerable error ratio with the same range of frequency ratio. From each group, we calculated the average results as shown in Figure 9. We plot the job latency, bandwidth utilization, and consumed energy on logarithmic scale marked in the left side while prediction and tolerable scale is plotted in normal scale marked in the right side of the figure. We see that as the frequency ratio increases, the job latency increases because more data needs to be processed, bandwidth utilization increases because more data needs to be fetched, consumed energy increases because more data needs to be collected, fetched and processed, and the prediction error decreases as more data is used for event prediction. Also, the tolerable error ratio is always $<1$. It confirms the effectiveness of data collection strategy on reducing job latency, bandwidth consumption, and energy consumption without violating the required error limit.

\section{CONCLUSION}

To enhance the extensive use of edge computing in important domains such as ICA, it is essential to design strategies to reduce job latency, bandwidth utilization, and energy consumption, which are three critical performance metrics in edge computing systems. State-of-the-art methods for edge computing fail to address this issue considering the duplicated computation and data in ICA applications. Our proposed Context-aware Data Operation System (CDOS) incorporates three data operation strategies: data sharing and placement, data collection, and redundancy elimination that enables the sharing of source data, intermediate results and final results among edge nodes, adaptive adjustment of data collection frequency to achieve an optimal tradeoff between performance and computation accuracy and eliminates redundant data in data transmission. Our extensive evaluation results on both simulation and real test-bed showed CDOS outperforms other methods in terms of job latency, bandwidth utilization, and energy consumption. In future, we will jointly consider job scheduling and data operations to further improve application performance.

\section{ACKNOWLEDGMENTS}

This research was supported in part by U.S. NSF grants NSF-1827674, CCF-1822965, FHWA grant 693JJ31950016, and Microsoft Research Faculty Fellowship 8300751.

\section{REFERENCES}

[1] Hossein Ahmadi, Tarek F. Abdelzaher, and Indranil Gupta. 2010. Congestion Control for Spatio-temporal Data in Cyber-physical Systems. In Proc. of ICCPS.

[2] P. K. Choubev, S. Pateria, A. Saxena, V. P. Chirayil SB, K. K. Jha, and S. Basaiah PM. 2015. Power Efficient, Bandwidth Optimized and Fault Tolerant Sensor Management for IOT in Smart Home. Proc. of IACC (2015).

[3] Byung-Gon Chun, Sunghwan Ihm, Petros Maniatis, Mayur Naik, and Ashwin Patti. 2011. CloneCloud: Elastic Execution Between Mobile Device and Cloud. In Proc. of EuroSys.

[4] Mingzhe Du, Yang Wang, and Chengzhong Xu. 2019. On Cost-Driven Computation Offloading in the Edge: A New Model Approach. Proc. of CCGRID (2019).

[5] Harshit Gupta, Amir Vahid Dastjerdi, Soumya K. Ghosh, and Rajkumar Buyya. 2017. iFogSim: A toolkit for modeling and simulation of resource management techniques in the Internet of Things, Edge and Fog computing environments. Software: Practice and Experience 47, 9 (jun 2017), 1275-1296.

[6] Shenghua He, Haiying Shen, Vivekgautham Soundararaj, and Lei Yu. 2018. Cloud Assisted Traffic Redundancy Elimination for Power Efficiency in Smartphones. Proc. of MASS (2018).

[7] Ke-Jou Hsu, Ketan Bhardwaj, and Ada Gavrilovska. 2019. Couper: DNN Model Slicing for Visual Analytics Containers at the Edge. In Proc. of SEC.

[8] Y. Huang, X. Song, F. Ye, Y. Yang, and X. Li. 2017. Fair Caching Algorithms for Peer Data Sharing in Pervasive Edge Computing Environments. In Proc. of ICDCS.

[9] H. Jeong, I. Jeong, H. Lee, and S. Moon. 2018. Computation Offloading for Machine Learning Web Apps in the Edge Server Environment. In Proc. of ICDCS.

[10] J. Lan, X. Liu, P. Shenoy, and K. Ramamritham. 2003. Consistency maintenance in peer-to-peer file sharing networks. Proc. of WIAPP (2003).

[11] Chunlin Li, Jingpan Bai, and Tang Jianhang. 2019. Joint optimization of data placement and scheduling for improving user experience in edge computing. 7 . Parallel Distrib. Comput. 125 (2019).

[12] Lin Wang, L. Jiao, D. Kliazovich, and P. Bouvry. 2016. Reconciling task assignment and scheduling in mobile edge clouds. In Proc. of ICNP.

[13] Guoxin Liu, Haiying Shen, and Haoyu Wang. 2016. Deadline Guaranteed Service for Multi-Tenant Cloud Storage. IEEE Trans. Parallel Distrib. Syst. (2016).

[14] L. Liu, Z. Chang, X. Guo, S. Mao, and T. Ristaniemi. 2018. Multiobjective Optimization for Computation Offloading in Fog Computing. IEEE Internet of Things Journal 5, 1 (Feb 2018).

[15] J. Meng, H. Tan, C. Xu, W. Cao, L. Liu, and B. Li. 2019. Dedas: Online Task Dispatching and Scheduling with Bandwidth Constraint in Edge Computing. In Proc. of INFOCOM.

[16] F. Messaoudi, A. Ksentini, and P. Bertin. 2017. On Using Edge Computing for Computation Offloading in Mobile Network. In Proc. of GLOBECOM.

[17] Mohammed Islam NAAS, Laurent Lemarchand, Jalil Boukhobza, and Philippe Raipin. 2018. A Graph Partitioning-based Heuristic for Runtime IoT Data Placement Strategies in a Fog Infrastructure. In Proc. of the ASAC. 
[18] Mohammed Islam Naas, Philippe Raipin Parvedy, Jalil Boukhobza, and Laurent Lemarchand. 2017. iFogStor: An IoT Data Placement Strategy for Fog Infrastructure. In Proc. of ICFEC.

[19] Chanh Nguyen, Amardeep Mehta, Cristian Klein, and Erik Elmroth. 2019. Why Cloud Applications Are Not Ready for the Edge (Yet). In Proc. of SEC.

[20] Tahmid Rashid, Daniel (Yue) Zhang, and Dong Wang. 2018. EdgeStore: Towards an Edge-based Distributed Storage System for Emergency Response. In Proc. of Smart City.

[21] X. Song, Y. Huang, Q. Zhou, F. Ye, Y. Yang, and X. Li. 2017. Content Centric Peer Data Sharing in Pervasive Edge Computing Environments. In Proc. of ICDCS.

[22] W. R. Stevens. 1994. TCP/IP Illustrated Volume 1. AddisonWesley (1994).

[23] Nathan Vance, Yue Zhang, and Dong Wang. 2019. EdgeCache: A game-theoretic edge-based content caching system for crowd video sharing. Proc. of HPCC (2019).

[24] Karima Velasquez, David Perez Abreu, Marilia Curado, and Edmundo Monteiro. 2017. Service placement for latency reduction in the internet of things. Annals of Telecommunications 72, 1 (01 Feb 2017).

[25] J. Xie, C. Qian, D. Guo, M. Wang, S. Shi, and H. Chen. 2019. Efficient Indexing Mechanism for Unstructured Data Sharing Systems in Edge Computing. In Proc. of INFOCOM.

[26] Lei Yang, Jiannong Cao, Yin Yuan, Tao Li, Andy Han, and Alvin Chan. 2013. A Framework for Partitioning and Execution of Data Stream Applications in Mobile Cloud Computing. SIGMETRICS Perform. Eval. Rev. 40, 4 (April 2013).
[27] L. Yu, H. Shen, K. Sapra, L. Ye, and Z. Cai. 2017. CoRE: Cooperative End-to-End Traffic Redundancy Elimination for Reducing Cloud Bandwidth Cost. IEEE Trans. on Parallel and Distributed Systems 28, 2 (2017).

[28] D. Zeng, L. Gu, S. Guo, Z Cheng, and S. Yu. 2016. Joint Optimization of Task Scheduling and Image Placement in Fog Computing Supported Software-Defined Embedded System. IEEE Trans. on Computers 65, 12 (Dec 2016).

[29] Daniel W. Zhang and Dong Wang. 2019. An Integrated Top-down and Bottom-up Task Allocation Approach in Social Sensing based Edge Computing Systems. Proc. of INFOCOM (2019).

[30] Daniel (Yue) Zhang, Tahmid Rashid, Xukun Li, Nathan Vance, and Dong Wang. 2019. HeteroEdge: Taming the Heterogeneity of Edge Computing System in Social Sensing. In Proc. of IoTDI.

[31] Xingzhou Zhang, Mu Qiao, Liangkai Liu, Yunfei Xu, and Weisong Shi. 2019. Collaborative Cloud-Edge Computation for Personalized Driving Behavior Modeling. In Proc. of SEC.

[32] Y. Zhang, D. Zhang, N. Vance, Q. Li, and D. Wang. 2018. A Light-Weight and Quality-Aware Online Adaptive Sampling Approach for Streaming Social Sensing in Cloud Computing. In Proc. of ICPADS

[33] Li Zhou, Mohammad Hossein Samavatian, Anys Bacha, Saikat Majumdar, and Radu Teodorescu. 2019. Adaptive Parallel Execution of Deep Neural Networks on Heterogeneous Edge Devices. In Proc. of SEC.

[34] Yan Zhuang, Lei Yu, Haiying Shen, William Kolodzey, Nematollah Iri, Gregori Caulfield, and Shenghua He. 2019. Data Collection with Accuracy-Aware Congestion Control in Sensor Networks. IEEE Trans. Mob. Comput. 18, 5 (2019). 